\title{
A localization algorithm based on AOA for ad-hoc sensor networks
}

\author{
Yang Sun Lee ${ }^{\mathrm{a}}$, Jang Woo Park ${ }^{\mathrm{b}}$ and Leonard Barolli ${ }^{\mathrm{c}, *}$ \\ ${ }^{a}$ Department of Information Communication Engineering, Chosun University, Chosun, Korea \\ ${ }^{\mathrm{b}}$ Department of Information and Communication Engineering, Sunchon National University, Sunchon, \\ Korea \\ ${ }^{\mathrm{c}}$ Department of Information and Communication Engineering, Fukuoka Institute of Technology (FIT), \\ Fukuoka, Japan
}

\begin{abstract}
Knowledge of positions of sensor nodes in Wireless Sensor Networks (WSNs) will make possible many applications such as asset monitoring, object tracking and routing. In WSNs, the errors may happen in the measurement of distances and angles between pairs of nodes in WSN and these errors will be propagated to different nodes, the estimation of positions of sensor nodes can be difficult and have huge errors. In this paper, we will propose localization algorithm based on both distance and angle to landmark. So, we introduce a method of incident angle to landmark and the algorithm to exchange physical data such as distances and incident angles and update the position of a node by utilizing multiple landmarks and multiple paths to landmarks.
\end{abstract}

Keywords: AoA, RSSI, wireless sensor network, localization

\section{Introduction}

Wireless sensor networks (WSNs) involve many different technologies such as communication, sensing and computing, which now becoming a very important research areas. The knowledge of the accurate locations of sensor nodes in WSN makes possible many attractive applications such as routing and tracking asset [1-4]. The position coordinates of sensor node in WSN can add significant meaning to information which are collected by sensors [5-7].

Sensor node location can be computed by Range based methods such as GPS (Global Positioning System) or Range-free method. The GPS-based method needs sensor nodes to be equipped by GPS which is very expensive and cannot be used in the case of blocking the GPS signal such as indoor environment.

The distance among sensor nodes can be determined by RSSI (Received Signal Strength Indicator), ToA/TDoA (Time of Arrival/ Time Difference of Arrival) and AOA (Angle of Arrival). If distances and angles between pairs of nodes can be measured accurately, the localization process will be very simple. But measurements can be with errors, so the localization algorithm can become complex and inaccurate.

There have been a lot of research on the positioning technologies [8-12]. Among these researches, DV-hop and DV-distance proposed by Niculescu $[8,9]$ have been well known. In this paper, it is assumed

\footnotetext{
${ }^{*}$ Corresponding author: Leonard Barolli, Department of Information and Communication Engineering, Fukuoka Institute of Technology (FIT), 3-30-1 Wajiro-Higashi, Higashi-Ku, Fukuoka 811-0295, Japan. E-mail: barolli@ fit.ac.jp.
} 
that WSN consists of sensor nodes and landmarks. The coordinates of landmarks can be given by GPS or directly by humans. Also, it is also assumed that the sensors have ability to measure the distance and relative angles to their neighbors. Sensors except landmarks don't know their absolute reference bearing such as north so that they should infer the reference bearing from the bearings of landmarks. In [13], we have already introduced a method to obtain the bearings of sensor nodes and conformed the effectiveness of our method by comparing with DV-hop and DV-distance. In this paper, we will give more detail positioning algorithm and some results with node densities, measuring physical data and the number of landmarks.

This paper is organized as follows. In Section 2, we present the related works. Then, in Section 3, we introduce in details the method to measure the angles to neighbors [13]. In Section 4, we present the positioning algorithm proposed in this paper in more details. In Section 5 are shown the simulation results. Finally, in the last Section, we give the concluding remarks.

\section{Related works}

Several localization technologies using wireless radios have been developed and commercially available. The GPS is perhaps the most popular location system but GPS cannot be used indoor or when receivers cannot receive the wireless signal from the satellites. To complement the weakness of GPS, there have been many available localization systems such as Active badge, Active bat, Cricket, and Radar and their detail review is found in [14].

The Active badge localization system was developed at Olivetti Research Laboratory, now AT\&T Cambridge and uses diffuse infrared technology. The Active badge system provides absolute location information and difficulty in locations with fluorescent lighting or direct sunlight. Because diffused infrared has an effective range of only several meters, Active badge system can be deployed in small or medium sized rooms.

Active bat localization system was developed by AT\&T. This system uses ultrasound time of flight technique so that it can provide more accurate measurements than Active Badges. A bat sends an ultrasonic pulse to a ceiling mounted receivers and at the same time the controller sends not only the radio frequency request packet but also a synchronized reset signal to the ceiling sensors. Each ceiling sensors measures the time interval between RF signal reset arrival and ultrasonic pulse arrival and then the time difference will be converted distance. Active Bat can obtain accuracy within $9 \mathrm{~cm}$ of the true position.

Cricket location support system was intended to complement the Active Bat system. Therefore, like the Active Bat system utilizes ultrasonic time-of-flight data and radio frequency synchronization signal. This system allows the objects to perform all their triangulation computations. Cricket uses the RF signal for synchronization as well as description of the available time domain. Cricket provides both the lateration and proximity techniques.

RADAR has been developed by Microsoft Research group and is based on the IEEE 802.11 WaveLAN wireless networking technology. RADAR can measure the signal strength and signal-to-noise ratio of signal which is used to compute $2 \mathrm{D}$ position within the building. This system needs only a few base stations and facilitates the same infrastructure providing the building's general purpose wireless networking. Wireless radio localization system operates by measuring radio signals between a set of landmarks (or beacons or fixed stations) and unknown nodes (or mobile stations) in WSN. In the localization system in WSN, nodes can calculate their own positions by using signals received from landmarks. 


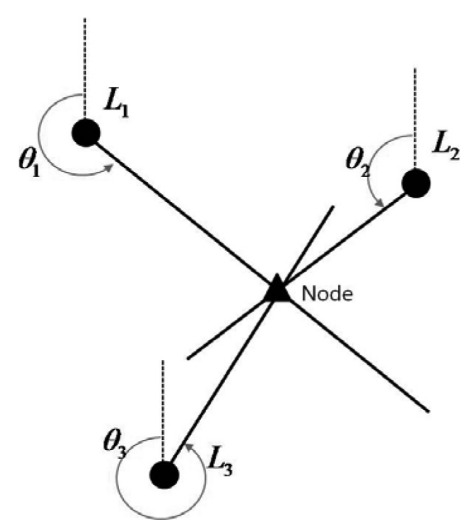

Fig. 1. Measuring the angles to determine the position of a node. Dots are landmarks which have already known their coordinates and triangle is a node to be located.

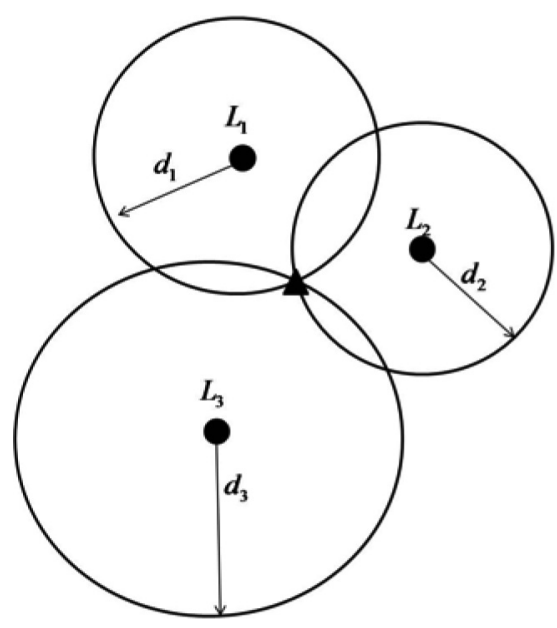

Fig. 2. Signal strength and ToA based localization system. Dots are landmarks which have already known their coordinates and triangle is a node to be located.

Wireless localization systems can be divided into angle based technique such as AoA, time based technique such as ToA and TDoA and signal strength based technique or their combinations. There is a brief review in [15].

Angle based techniques: AoA is the localization method based on Angle based techniques. Nodes in AoA techniques can estimate their locations by first measuring angles of received signals from several landmarks shown in Fig. 1. Measuring the AoA in nodes is available using directive antennas or antenna arrays. As shown in Fig. 1, finding the intersections of the lines-of-position allows the nodes to calculate their locations. AoA method needs a minimum of two landmarks to fix a position of a node.

Signal strength: The signal strength technique uses a known mathematical model describing the path loss attenuation with distance. The measured signal strength can convert the distance between a landmark and a node. A node to be located should be on a circle centered at the landmarks. As shown in Fig. 2, when measuring the signal strength from more than three landmarks, a node can find its location by using simple geometric relationships.

Time based localization: In ToA method, the distance between a landmark and a node can be found 


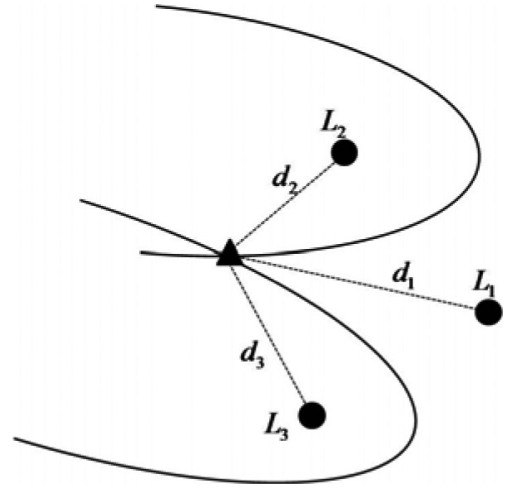

Fig. 3. TDoA based localization method. Dots are landmarks which have already known their coordinates and triangle is a node to be located.

by measuring one way propagation time between them assuming that the signals travel with the velocity of light (c). As shown in Fig. 2, if a node can know more than three distances to their neighboring landmarks, a node can locate its own position. The other hand, TDoA uses time differences of arrival signals. TDoA does not need to know the transmission time of a signal, which is important in ToA system. In TDoA technique, a line-of-position will be defined as hyperbola because a curve of constant time difference of arrivals for two landmarks is hyperbola as shown in Fig. 3.

\section{The method to measure the angle to immediate nodes}

WSN means the set of sensor nodes which are deployed ad-hoc. All nodes in WSN will be assumed to be possible to communicate with their neighboring nodes within their transmission range [16]. Especially the landmarks have already known their coordinates and they had their own reference bearings (for example, East). It will be assumed that all nodes have ability to measure the distance to their neighbors and relative angles based on their own axis (called heading). Figure 4 shows definitions of angles used in this paper [13]. The node's heading which is used for measuring the angle to neighbors is different among nodes. Node's headings are shown by thick arrows. In Fig. $4, \hat{a b}$ is the measured angle between node $\mathrm{A}$ and node C. $\theta$ shows the incident angle from neighbors with reference of East. For example, $\theta_{A B}$ is the incident angle from node A measured at node B. Then, $\hat{a}$ is the azimuth of node A which means the angle of A's heading measured from East.

Because the landmarks have their azimuths, the calculations will be started from the nodes near landmarks. First of all, assume that node B has been aware of its own azimuth and the angle to node A. Node $\mathrm{B}$ is able to calculate the incident angle, $\theta_{B A}$ using its own azimuth and the angle to node $\mathrm{A}$,

$$
\theta_{B A}= \begin{cases}\hat{b a}-\hat{b} & \text { for } \quad \hat{b a} \geqslant \hat{b} \\ 2 \pi+(\hat{b a}-\hat{b}) & \text { for } \quad \hat{b a}<\hat{b}\end{cases}
$$

Figure 4 (a) is for $\hat{b a}-\hat{b}$ and Fig. 4 (b) is for $\hat{b a} \geqslant \hat{b}$. The obtained incident angle $\theta_{B A}$ will be transferred to node $\mathrm{A}$ and then will be used for calculating the angles related to node $\mathrm{A}$. That is, node $\mathrm{A}$ will calculate the incident angle $\theta_{A B}$ from node $\mathrm{B}$ as follows,

$$
\theta_{A B}=2 \pi-\theta_{B A} .
$$




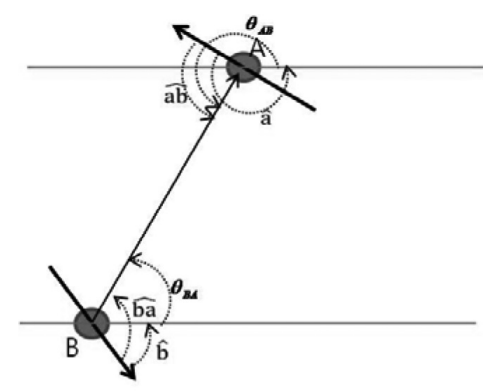

(a)

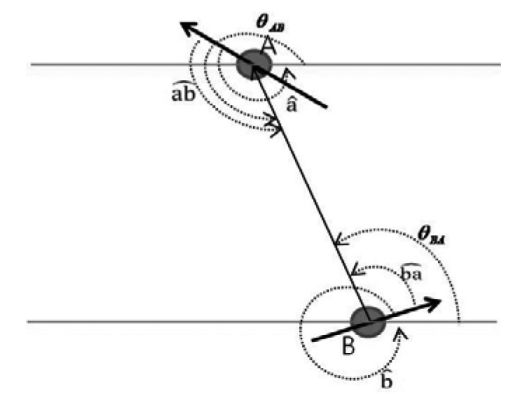

(b)

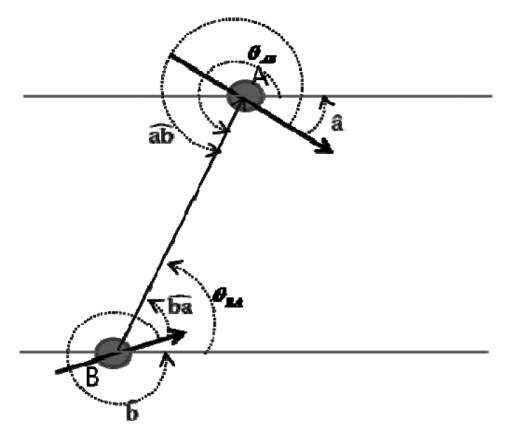

(c)

Fig. 4. Detail description for calculating useful angles between tow nodes (a) $\hat{b a} \geqslant \hat{b}$ and $\hat{b a}<180$, (b) $\hat{b a}<\hat{b}$, (c) $\hat{b a} \geqslant \hat{b}$ and $\hat{b a}>180$.

Also from $\theta_{A B}$ of Eq. (2) and the measured angle between node $\mathrm{B}$ and node $\mathrm{A}, \hat{a b}$, the azimuth of node A can be obtained,

$$
\hat{a}=\left\{\begin{array}{lll}
\hat{a} b-\theta_{A B} & \text { for } & \hat{a b} \geqslant \theta_{A B} \\
2 \pi+\left(\hat{a b}-\theta_{A B}\right) & \text { for } & \hat{a b} \leqslant \theta_{A B}
\end{array}\right.
$$

The same calculation will be performed for all nodes from the neighbors of landmarks so that all nodes with any connection to landmarks can have their own azimuths. And then nodes will also calculate all incident angles from their neighbors from their azimuths and the measured angles to their neighbors. Although nodes with at least one connection path to landmarks is possible to calculate the information related to angles, for nodes far from many hops from landmarks angle error accumulation will be profound. So, we will restrict the hop counts within 3 or 5 .

\section{Localization algorithm}

The localization algorithm proposed in this paper consists of two phases. For distribution process, these two phases will be performed simultaneously. In the first phase, all sensor nodes in the sensor field will find the neighbor nodes within their own transmission ranges. And then they have to calculate the distances between them and their neighbors and the incident angles from their neighbors. This physical 


\begin{tabular}{|c|c|c|c|}
\hline id & distance & theta & $\mathrm{L}$ or $\mathrm{S}$ \\
\hline
\end{tabular}

Fig. 5. Table for physical information.

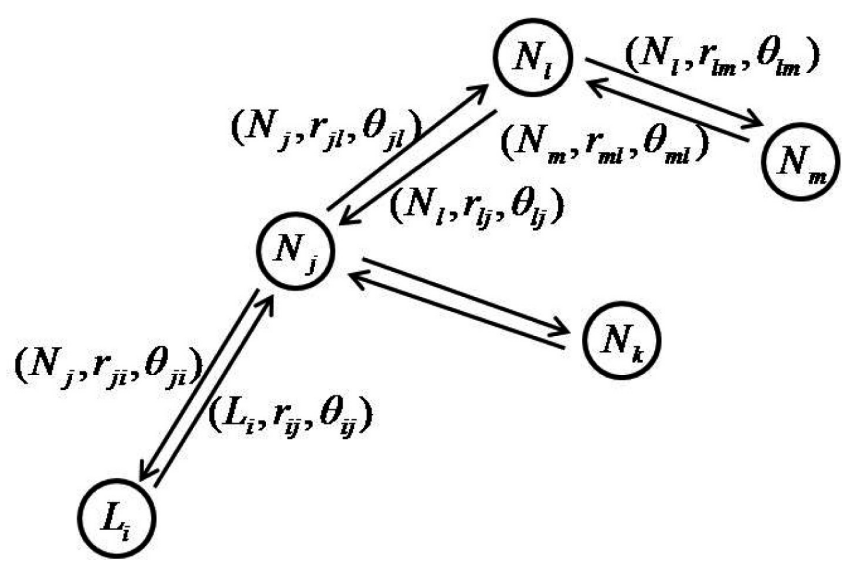

Fig. 6. The example for showing the data exchanges between nodes.

information will be recalculated and shared with their neighbors periodically or when the events happen such as adding new nodes. Each node will construct its physical information table as shown in Fig. 5.

In Fig. 5, the id is the identification numbers of neighbor nodes, distances means the distances between a node and its neighbors. These distances divide the distance calculated by a sensor node itself and the distance sent by its neighbors. "Theta" is the calculated incident angles which are composed by the value calculated by a node itself and the values sent by its neighbors. To identify whether the neighbors are landmarks or ordinary nodes, "L or S" will be recorded.

Figure 6 shows the example of the process by which sensor nodes exchanges the physical data. Each node will send the message including physical data such as their identification number, the distance from itself to their proximities, the incident angle calculated by the method described in previous chapter. So, after finishing this process, all nodes can share all physical information to calculate their positions with their neighbors. For example, in Fig. 3, node $N_{j}$ can construct its own physical table shown in Table 1.

A second phase will begin at landmarks, based on the physical data recoded in the physical data table. Firstly, since landmarks have known their position, they will broadcast their identification number and their coordinates. And then, the neighboring nodes of landmarks can calculate their positions from the stored physical data and the coordinates of their landmarks. For example, a node $N_{j}$ adjacent to the landmark $L_{i}$ in Fig. 6 can obtain its coordinate as follows

$$
\left(\begin{array}{l}
x_{j} \\
y_{j}
\end{array}\right)=\left(\begin{array}{l}
x_{i} \\
y_{i}
\end{array}\right)+\left(\begin{array}{c}
r_{j i} \cos \left(\theta_{j i}\right) \\
r_{j i} \sin \left(\theta_{j i}\right)
\end{array}\right)
$$

After the nodes have calculated its coordinate, they construct their position table which includes their coordinates and the hop counts, the hop numbers far from the landmarks which used in calculating the positions. The nodes which have had their positions are going to broadcast their coordinates to their neighbors and then the nodes received the position information from their neighbors can calculate their position. The nodes that have calculated their coordinates can make their position tables. This process will continue until all nodes will have their positions and will also take place when their neighbors' positions update. 
Table 1

The physical data table for node $N_{j}$

\begin{tabular}{ccccccc}
\hline Node id & \multicolumn{2}{c}{ Distances } & & \multicolumn{2}{c}{ Incident angles } & \multirow{2}{*}{ or S } \\
\cline { 2 - 3 } \cline { 5 - 6 } & From neighbors & By itself & & From neighbors & By itself & \\
\hline$L_{i}$ & $r_{i j}$ & $r_{j i}$ & & $\theta_{i j}$ & $\theta_{j i}$ & $\mathrm{~L}$ \\
$N_{k}$ & $r_{l j}$ & $r_{j l}$ & & $\theta_{l j}$ & $\theta_{j l}$ & $\mathrm{~S}$ \\
$N_{l}$ & $r_{k j}$ & $r_{j k}$ & & $\theta_{k j}$ & $\theta_{j k}$ & $\mathrm{~S}$ \\
\hline
\end{tabular}

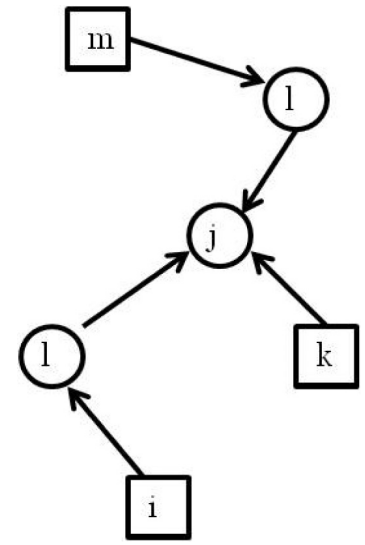

(a)

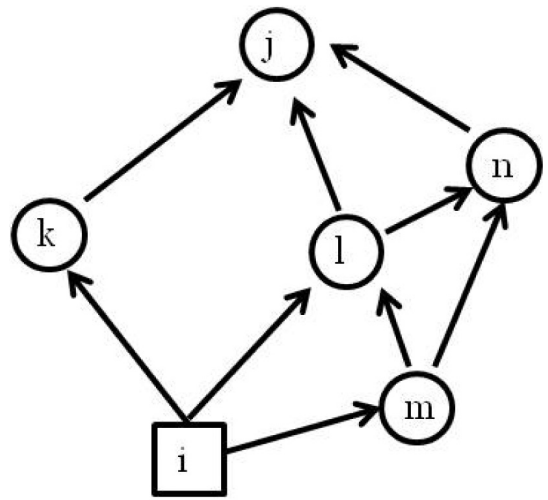

(b)

Fig. 7. In (a) sensor node $\mathrm{j}$ sees several landmarks; (b) there are several paths from landmark $\mathrm{i}$ to sensor node $\mathrm{j}$.

\subsection{Updating of coordinates of sensor nodes}

As some nodes could have known several landmark's coordinates or there are many paths from landmarks to nodes which want to know their positions, a sensor node may have several coordinates. Figure 7 shows the situation that a node has linked several landmarks and had several paths to a landmark. In Fig. 7 squares are the landmarks and circles are sensor nodes. In the case of Fig. 7 (a), sensor node $\mathrm{j}$ knows 2 landmarks, so it is possible for this node to have three coordinates. Figure 7 (b) shows there are several paths from landmark $i$ to sensor node $j$. In this case, the sensor node $j$ will have several coordinates for itself. This means it is necessary for nodes to have a method to determine their positions among several coordinates.

This paper proposes four schemes to determine the sensor's coordinate. The first scheme is what we called "min-hop" method. This scheme can choose the landmark minimum hops away from a sensor node and then sensor node come to calculate its coordinate from the position of this landmark. However, there are multiple landmarks same hops away; the position of the sensor node will be averaged. The second scheme is called "min-distance" method. First of all, the sensor node estimates the distance to known landmarks and then choose the closest landmark in distance. Accordingly, the sensor node will determine its position calculated from the closest landmark.

The third method utilizes the several coordinates calculated from several landmarks. If there are several paths from a sensor node to a landmark, we choose the path with minimum hops. So, this method averages the coordinates from several landmarks to determine the node's coordinate. We can see that in Fig. 7 (a) the first method uses only landmark k to find the coordinate of node $j$ but the third method utilizes all coordinates calculated from landmark i, $\mathrm{k}$ and $\mathrm{m}$. We call this method "averaged min-hop" method. 


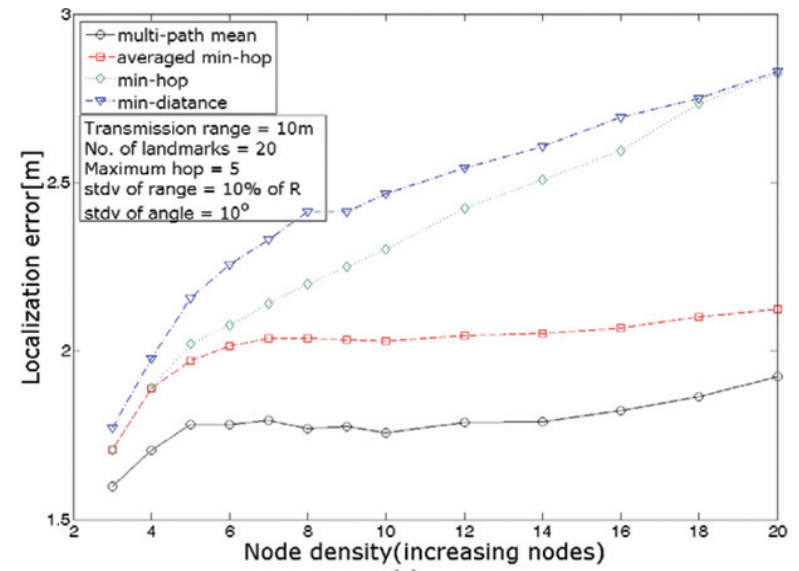

(a)

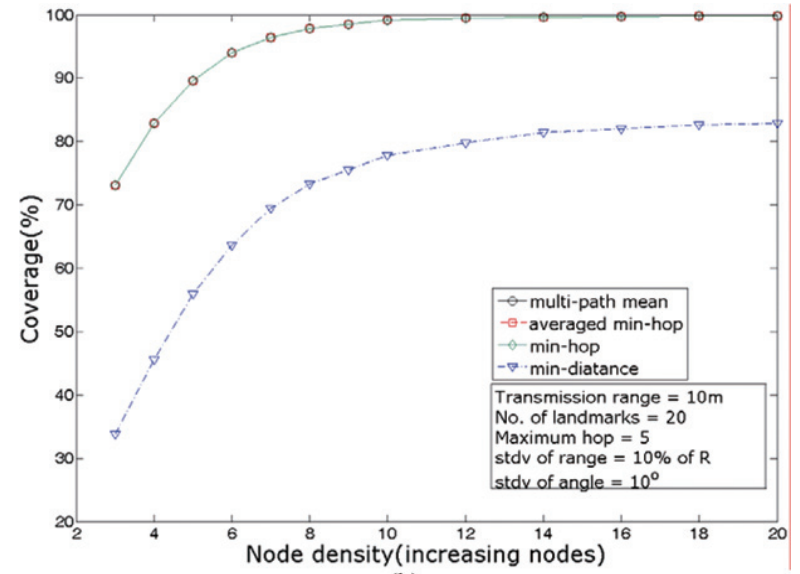

(b)

Fig. 8. Localization error and coverage with the variation of node density (node density is calculated by changing the number of nodes).

The final scheme is very simple. This method utilizes several paths from a landmark to a sensor node. As seen in Fig. 7 (b), there are multiple paths from a landmark to a node. This means a node can obtain several coordinates even when it knows only one landmark because several paths means several coordinates for a node. So, we will simply average the node's coordinates obtained from multiple path, so called "multi-path mean" method.

\section{Results and discussion}

The proposed method will be susceptible to angle error because the node's azimuth is calculated from a landmark far apart. This becomes severe when a landmark is far apart from. In this paper, the measured distance and angle are assumed to be Gaussian.

$$
\begin{aligned}
& r_{\text {meas }}=r_{\text {exact }}\left(1+\sigma_{r} N(0,1)\right) \\
& \theta_{\text {meas }}=\theta_{\text {exact }}\left(1+\sigma_{r} N(0,1)\right)
\end{aligned}
$$

where $r_{\text {meas }}\left(\theta_{\text {meas }}\right)$ is the measured distance (angle), $r_{\text {exact }}\left(\theta_{\text {exact }}\right)$ is the true value of the distance(angle), $\sigma_{r}\left(\sigma_{\theta}\right)$ is a specific constant [17], and $N(0,1)$ is a normally distributed random variable. Therefore, the noise error in measured values is modeled as additive and can be varied by changing the specific constants $\sigma_{r}\left(\sigma_{\theta}\right)$, where in Matlab ${ }^{\circledR}$ program, $\sigma_{r}\left(\sigma_{\theta}\right)$ in Eqs (5), (6) are the standard deviation of normal distribution, so we will simply call it a standard deviation.

Figure 8 shows the location error and coverage with the node density, where coverage means the ratio of nodes calculating the coordinate to all nodes. Node density [18-20] can be calculated as,

$$
d=\frac{N\left(\pi R^{2}\right)}{A}
$$

where $\mathrm{N}$ is the total number of sensor nodes deployed in sensor field, $\mathrm{A}$ is the area of sensor field, $\mathrm{R}$ means the transmission range of nodes which is the same among all nodes. Localization error obtained 


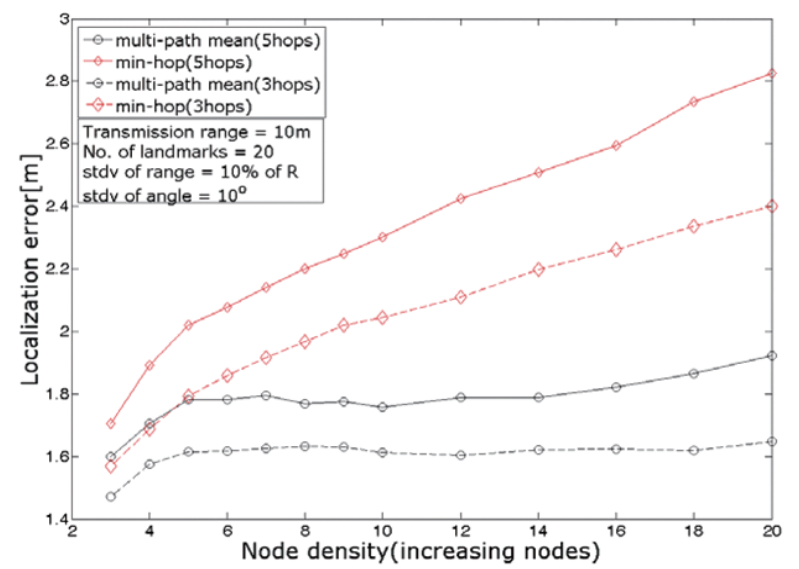

(a)

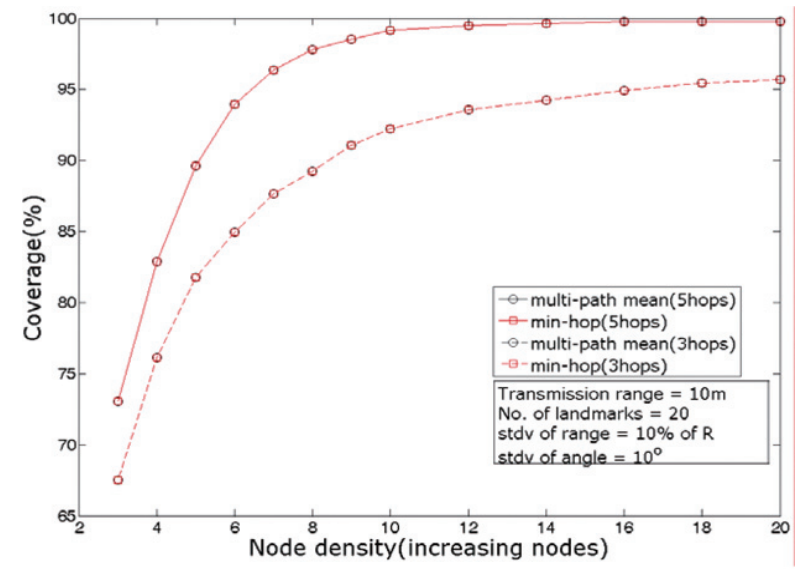

(b)

Fig. 9. Comparing the results between limited maximum hops of 3 and 5. (a) Accuracy (b) Coverage.

in the simulation is defined by:

$$
L_{\text {error }}=\frac{\sum_{i=1}^{N}\left|r_{\text {calc }}-r_{\text {real }}\right|}{N}
$$

where $\mathrm{N}$ is the total number of nodes, $r_{\text {calc }}$ is the calculated coordinate of a node, and $r_{\text {real }}$ is the real coordinate of a node.

In this simulation, sensor field is considered to be square of which length of each side is $100 \mathrm{~m}$. The transmission range is $10 \mathrm{~m}$. As shown in Eq. (7), the node density can be changed according to varying the number of nodes or transmission range when the area of sensor field is fixed. The results in Fig. 8 are obtained from varying the number of nodes. In this simulation, we restrict the hop count to landmarks within 5 in order to prevent the propagation of angle error. That is, when calculating the coordinates of nodes, only landmarks within 5 hops far from a node were considered. The larger in the hop counts, the larger coverage is but the larger localization error becomes. The "multi-path mean" method reveals the best result and the "averaged min-hop" method ranks second in the accuracy. Also, two methods are nearly independent upon the node density but the "min-hop" and "min-distance" method show that the accuracies increase with the increasing of the node density. In the aspect of the coverage, three methods except "min-distance" method show the same result. That is, the coverage obtained from our method reaches nearly $100 \%$ when the node density of about 10 .

To see the effect of measuring errors in distance and angle on the accuracy and coverage, two results obtained with allowed maximum hops of 3 and 5 are compared in Fig. 9. In Fig. 9, the solid lines show the results for maximum hop limited to 5 and dashed line are the result for 3 hops. The accuracy in the case of allowed maximum hop of 3, in Fig. 9(a), is better than that of 5 hops in the expense of coverage shown in Fig. 9(b). The coverage of 5 hops reaches nearly $100 \%$ for node density of 12 . However, in the case of maximum hop of 3 , it reaches about $90 \%$.

The effect of increasing the number of landmarks on the localization error and coverage is shown in Fig. 10. In general, increasing the number of landmarks can make hops to a node small so that the accumulation of angle error can be reduced, which allows the node to choose the landmark with the small error. When the number of landmarks are smaller than 40, the "multi-path mean" method shows the best accuracy, but with landmarks more than 40 , the "averaged min-hop" method reveals better accuracy than 




(a)

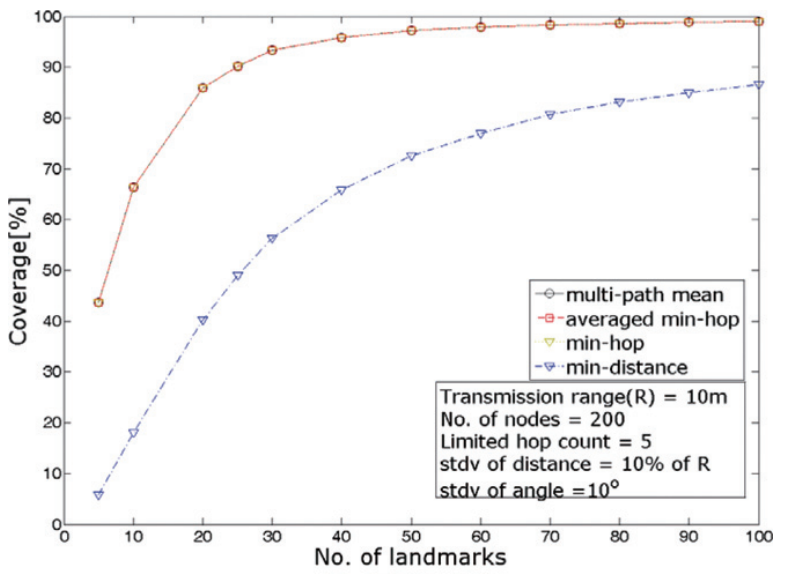

(b)

Fig. 10. Localization error and coverage with the number of landmarks.

other methods. In this simulation, because we restrict the hop counts to 5 to find landmarks, there are still isolated nodes from landmarks even though increasing the number of landmarks so that coverage will not reach $100 \%$.

\section{Conclusions}

We introduced a localization algorithm to find the coordinates of nodes in WSN. This method assumed that the nodes have the ability to measure the mutual distance and relative angle to their neighbors within transmission range. The proposed algorithm starts at measuring the angle and distance at the landmark and their neighbors and then finding their azimuths and incident angles. Obtained incident angle and measured mutual distance allow a node to calculate its coordinates.

In this paper, we presented detailed description for utilizing multiple landmarks' coordinates and data from multiple paths. We presented four kinds of methods to calculate and update the coordinates of nodes. In "min-hop" method, the landmark is selected by minimum number of hops and the sensor node calculates its coordinates from the position of this landmark. The second method is called "min-distance" method where the sensor node estimates the distance to known landmarks and then chooses the closest landmark considering the distance. Therefore, the sensor node will determine its position calculated from the closest landmark. The third method utilizes several coordinates calculated from several landmarks, where the coordinates from several landmarks to determine the node's coordinate are simply averaged. This method is called "averaged min-hop" method. The last scheme, which is called "multi-path mean" method, utilizes several paths from a landmark to a sensor node when there are multiple paths from a landmark to a node. This method will simply average the node's coordinates obtained from multiple paths. The simulation results show that to utilize more data obtained from multiple paths can give more accurate position coordinates.

\section{Acknowledgements}

This work was supported by the National Research Foundation of Korea(NRF) grant funded by the Korea government(MEST) (No. 2010-0013411). 


\section{References}

[1] A. Barolli, E. Spaho, L. Barolli, F. Xhafa and M. Takizawa, QoS Routing in Ad-hoc Networks Using GA and Multiobjective Optimization, Mobile Information Systems 7(3) (2011), 169-188.

[2] W. Wu, X. Li, S. Xiang, H.B. Lim and K.L. Tan, Sensor Relocation for Emergent Data Acquisition in Sparse Mobile Sensor Networks, Mobile Information Systems 6(2) (2010), 155-176.

[3] J. Jayaputera and D. Taniar, Data Retrieval for Location-dependent Queries in a Multi-cell Wireless Environment, Mobile Information Systems 1(2) (2005), 91-108.

[4] A.B. Waluyo, B. Srinivasan and D. Taniar, Research on Location-dependent Queries in Mobile Databases, International Journal of Computer Systems: Science and Engineering 20(2) (2005), 77-93.

[5] P. Sarkar and A. Saha, Security Enhanced Communication in Wireless Sensor Networks Using Reed-Muller Codes and Partially Balanced Incomplete Block Designs, Future Technology Research Association International (FTRA), Journal of Convergence 2(1) (2011), 43-48.

[6] C.M. Huang, R.H. Cheng, S.R. Chen and C.I. Li, Enhancing Network Availability by Tolerance Control in Multi-Sink Wireless Sensor Networks, FTRA Journal of Convergence 1(1) (2010), 15-22.

[7] D. Kumar, C. Trilok, R. Aseri and B. Patel, Multi-hop Communication Routing (MCR) Protocol for Heterogeneous Wireless Sensor Networks, International Journal of Information Technology, Communications and Convergence 1(2) (2011), 130-145.

[8] D. Niculescu and B. Nath, Ad-hoc Positioning System (APS), Proc. of IEEE Global Telecommunications Conference (GLOBECOM'01) 5 (2001), 2926-2931.

[9] D. Niculescu and B. Nath, Ad Hoc Positioning System (APS) Using AOA, Proc. of the 23-rd Conference of the IEEE Communications Society (INFOCOM'03) (2003), 1734-1743

[10] G.D. Stefano and A. Petricola, A Distributed AOA Based Localization Algorithm for Wireless Sensor Networks, Journal of Computers 3(4) (2008), 1-8.

[11] A. Savvides, C. Han and M.B. Strivastava, Dynamic Fine-Grained Localization in Ad-hoc Networks of Sensors, Proc. of MobCom-2001 (2001), 166-179.

[12] M. Boushaba, A. Hafid and A. Benslimane, High Accuracy Localization Method using AOA in Sensor Networks, Computer Networks 53(18) (2009), 3076-3088.

[13] J. Park and D. Park, Ad-Hoc Localization Method Using Ranging and Bearing, To appear in Proc. of International Conference on Information Technology Convergence and Services (ITCS-2011) (2011).

[14] J. Hightower and G. Borriello, Location Systems for Ubiquitous Computing, IEEE Computer (2001), 57-66.

[15] J. Gibson, The Mobile Communication Handbook, IEEE Press (1999).

[16] B. Xie, A. Kumar, D. Zhao, R. Reddy and B. He, On Secure Communication in Integrated Heterogeneous Wireless Networks, International Journal of Information Technology, Communications and Convergence 1(1) (2010), 4-23.

[17] P. Biswas, H. Aghajan and Y. Ye, Integration of Angle of Arrival Information for Multimodal Sensor Network Localization using Semidefinite Programming, Proc. of 39-th Asilomar Conference on Signals, Systems and Computers (2005), 1-9.

[18] K.K. Chintalapudi, A. Dhariwal, R. Govindan and G. Sukhatme, Ad-Hoc Localization Using Ranging and Sectoring, Proc. of the 23-rd Conference of the IEEE Communications Society (INFOCOM-2004) (2004), 2662-2672.

[19] K.K. Chintalapudi, A. Dhariwal, R. Govindan and G. Sukhatme, On the Feasibility of Ad-Hoc Localization Systems, Tech. Report, Computer Science Department, University of Southern California, Los Angeles (2003).

[20] K. Liu, S. Wang, F. Zhang, F. Hu and C. Xu, Efficient Localized Localization Algorithm for Wireless Sensor Networks, Proc. of the Fifth International Conference on Computer and Information Technology (CIT-2005) (2005), 517-523.

Yang Sun Lee received the B.S. and M.S. degrees in Electrical \& Electronic Engineering from Dongshin University and Ph. D. degrees in Dept. of IT Engineering from Mokwon University in 2001, 2003 and 2007, respectively. He was a Senior Engineer at R\&D Center, Fumate Co., Ltd. from 2007 to 2009. Since 2009, he has worked as a Research Professor in Dept. of Information Communication Engineering at Chosun University. His current research interests include UWB, multimedia communication, Network Transmission Scheme and Ubiquitous Sensor Networks. He is a member of the KICS, KIMICS, KEES, KIIT and KONI.

Jang Woo Park received the B.S., M.S., and Ph.D. degrees in Electronic engineering from Hanyang University, Seoul, Korea in 1987, 1989 and 1993 respectively. In 1995, he joined the faculty member of the Sunchon National University, where he is currently a professor in the Dept. of Computer \& Communication Engineering. His research focuses on RFID circuits and system designs and RFID/USN technologies.

Leonard Barolli received B.S and Ph.D degrees from Tirana University and Yamagata University in 1989 and 1997, respectively. From April 1997 to March 1999, he was a JSPS Post Doctor Fellow Researcher at Department of Electrical and Information 
Engineering, Yamagata University. From April 1999 to March 2002, he worked as a Research Associate at the Department of Public Policy and Social Studies, Yamagata University. From April 2002 to March 2003, he was an Assistant Professor at Department of Computer Science, Saitama Institute of Technology (SIT). From April 2003 to March 2005, he was an Associate Professor and presently is a Full Professor, at Department of Information and Communication Engineering, Fukuoka Institute of Technology (FIT). Dr. Barolli has published about 350 papers in referred Journals, Books and International Conference Proceedings. He was an Editor of the IPSJ Journal and has served as a Guest Editor for many International Journals. Dr. Barolli has been a PC Member of many International Conferences and was the PC Chair of IEEE AINA-2004 and IEEE ICPADS-2005. He was General Co-Chair of IEEE AINA-2006, AINA-2008, AINA-2010, CISIS-2009, CISIS-2010, and CISIS-2011, Workshops Chair of iiWAS-2006/MoMM-2006 and iiWAS-2007/MoMM-2007, Workshop Co-Chair of ARES2007, ARES-2008, IEEE AINA-2007 and ICPP-2009. Dr. Barolli is the Steering Committee Chair of CISIS and BWCCA International Conferences and is serving as Steering Committee Co-Chair of IEEE AINA, IEEE INCoS, NBiS, 3PGCIC and EIDWT International Conferences. He is organizers of many International Workshops. Dr. Barolli has won many Awards for his scientific work and has received many research funds. He got the "Doctor Honoris Causa" Award from Polytechnic University of Tirana in 2009. His research interests include network traffic control, fuzzy control, genetic algorithms, P2P, wireless networks, ad-hoc networks and sensor networks. He is a member of SOFT, IPSJ, and IEEE. 

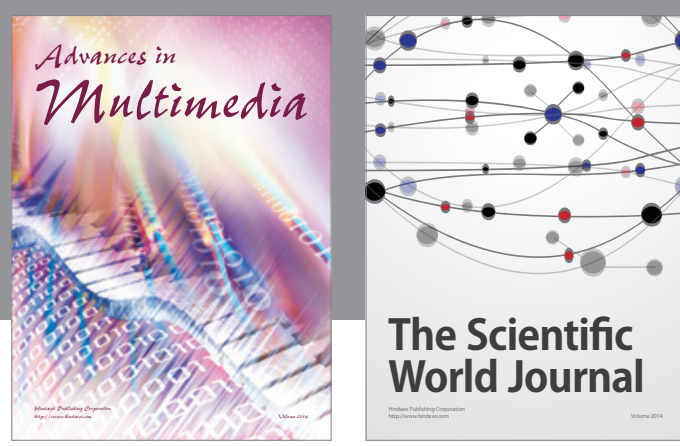

The Scientific World Journal
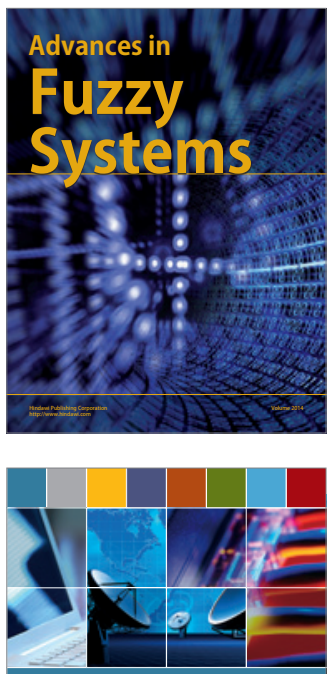

Computer Networks and Communications

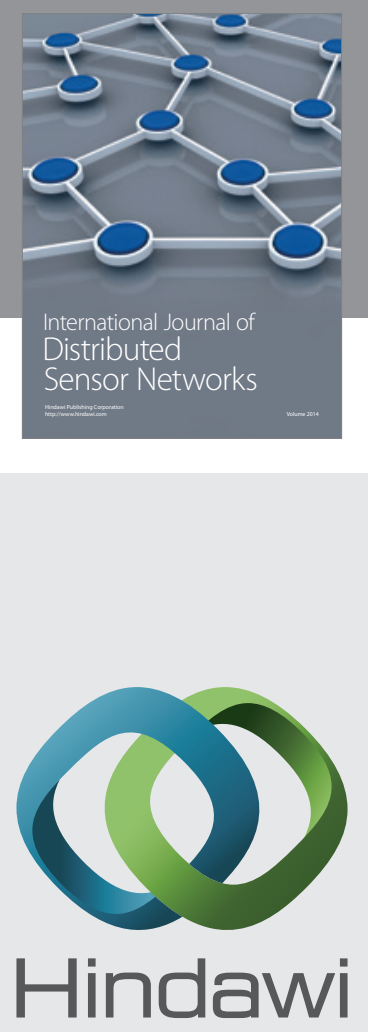

Submit your manuscripts at

http://www.hindawi.com
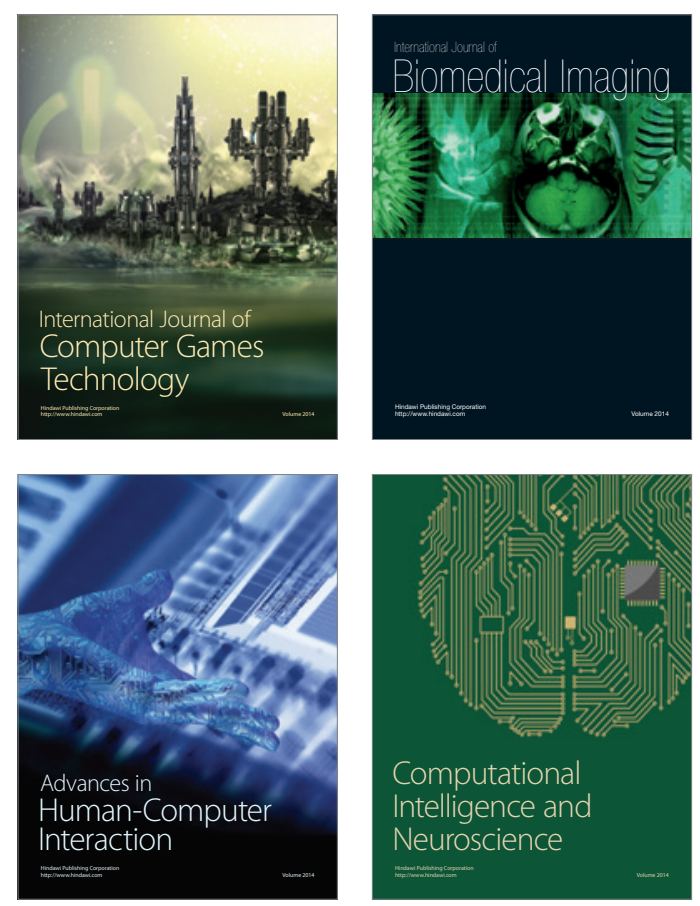
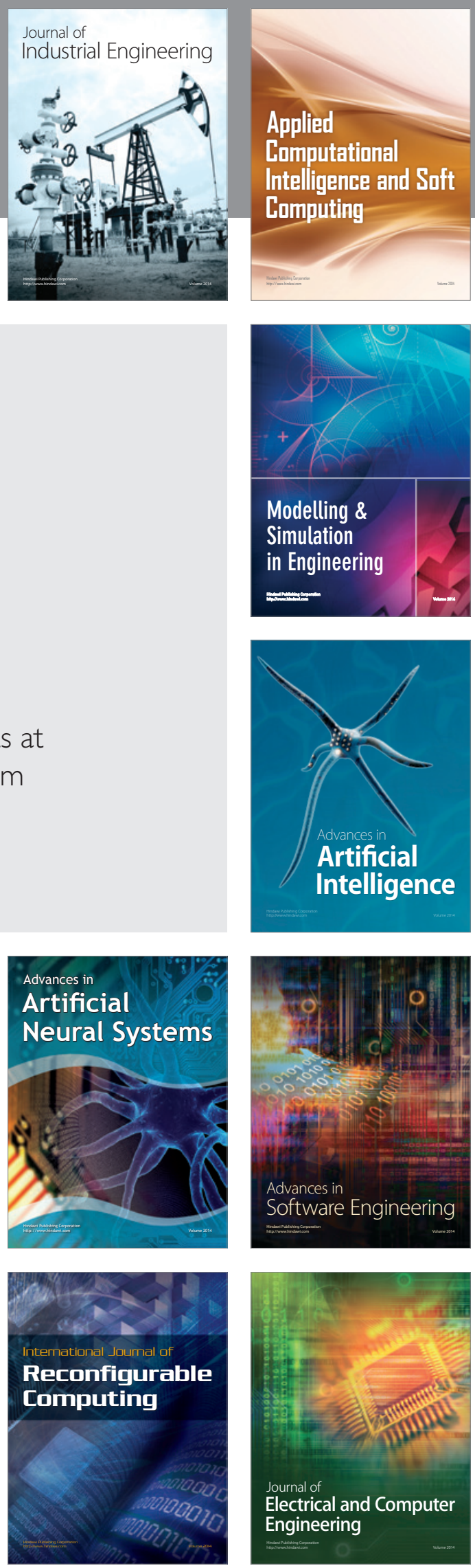